\title{
Characterising cognitive heterogeneity in individuals at clinical high-risk for psychosis: a cluster analysis with clinical and functional outcome prediction
}

\author{
Kate Haining ${ }^{1} \cdot$ Ruchika Gajwani $^{2} \cdot$ Joachim Gross ${ }^{1,3} \cdot$ Andrew I. Gumley $^{2} \cdot$ Robin A. A. Ince $^{1} \cdot$ Stephen M. Lawrie ${ }^{4}$. \\ Frauke Schultze-Lutter $^{5,6,7} \cdot$ Matthias Schwannauer $^{8} \cdot$ Peter J. Uhlhaas $^{1,9}$
}

Received: 11 February 2021 / Accepted: 26 July 2021 / Published online: 16 August 2021

(c) Crown 2021, corrected publication 2021

\begin{abstract}
Schizophrenia is characterised by cognitive impairments that are already present during early stages, including in the clinical high-risk for psychosis (CHR-P) state and first-episode psychosis (FEP). Moreover, data suggest the presence of distinct cognitive subtypes during early-stage psychosis, with evidence for spared vs. impaired cognitive profiles that may be differentially associated with symptomatic and functional outcomes. Using cluster analysis, we sought to determine whether cognitive subgroups were associated with clinical and functional outcomes in CHR-P individuals. Data were available for 146 CHR-P participants of whom 122 completed a 6- and/or 12-month follow-up; 15 FEP participants; 47 participants not fulfilling CHR-P criteria (CHR-Ns); and 53 healthy controls (HCs). We performed hierarchical cluster analysis on principal components derived from neurocognitive and social cognitive measures. Within the CHR-P group, clusters were compared on clinical and functional variables and examined for associations with global functioning, persistent attenuated psychotic symptoms and transition to psychosis. Two discrete cognitive subgroups emerged across all participants: 45.9\% of CHR-P individuals were cognitively impaired compared to $93.3 \%$ of FEP, $29.8 \%$ of CHR-N and $30.2 \%$ of HC participants. Cognitively impaired CHR-P participants also had significantly poorer functioning at baseline and follow-up than their cognitively spared counterparts. Specifically, cluster membership predicted functional but not clinical outcome. Our findings support the existence of distinct cognitive subgroups in CHR-P individuals that are associated with functional outcomes, with implications for early intervention and the understanding of underlying developmental processes.
\end{abstract}

Keywords Clinical high-risk · First-episode psychosis · Cognition · Cluster analysis · Heterogeneity · Functional outcome

\section{Introduction}

Schizophrenia is a debilitating psychiatric disorder characterised by psychotic symptoms, including hallucinations and delusions, as well as impairments in cognition, sensory

Peter J. Uhlhaas

peter.uhlhaas@charite.de

1 Institute of Neuroscience and Psychology, University of Glasgow, Glasgow, UK

2 Institute of Health and Wellbeing, University of Glasgow, Glasgow, UK

3 Institute for Biomagnetism and Biosignalanalysis, University of Münster, Münster, Germany

4 Department of Psychiatry, University of Edinburgh, Edinburgh, UK processing and psychosocial functioning [1, 2]. Cognitive impairments span several domains including processing speed, working memory, executive functions, attention and social cognition [3, 4]. Schizophrenia is preceded, in the majority of cases, by a clinical high-risk for psychosis
5 Department of Psychiatry and Psychotherapy, Medical Faculty, Heinrich Heine University, Düsseldorf, Germany

6 Department of Psychology and Mental Health, Faculty of Psychology, Airlangga University, Surabaya, Indonesia

7 University Hospital of Child and Adolescent Psychiatry and Psychotherapy, University of Bern, Bern, Switzerland

8 Department of Clinical Psychology, University of Edinburgh, Edinburgh, UK

9 Department of Child and Adolescent Psychiatry, Charité Universitätsmedizin, Berlin, Germany 
(CHR-P) state lasting approximately 5-6 years [5]. CHR-P status is determined using ultra-high-risk (UHR) criteria, encompassing attenuated psychotic symptoms (APS), brief frank psychosis and functional decline with genetic risk [6], as well as basic symptom criteria that involve self-experienced perceptual and cognitive disturbances [7, 8]. CHR-P individuals are also characterised by widespread cognitive impairments intermediate between healthy controls (HC) and first-episode psychosis (FEP) patients $[9,10]$. These impairments, especially in attention, working memory and declarative memory, are more pronounced in CHR-P individuals who later transition to psychosis [11]. However, cognitive performance within the CHR-P state is highly variable with small-to-large effect size impairments (Cohen's $d=-0.35$ to -0.84$)$ in those who transition to psychosis and small-to-medium impairments $(d=-0.26$ to -0.67$)$ in those who do not [9]. Accordingly, novel approaches may be required to identify subtypes of CHR-P participants with different cognitive profiles, with possible implications for the understanding of underlying pathophysiology and accurate prediction of outcomes.

Data-driven approaches, such as cluster analysis, classify individuals according to levels and patterns of performance, rather than pre-determined grouping criteria [12]. Cognitive subgroups have successfully been identified in crossdiagnostic samples, comprising individuals with schizophrenia-spectrum disorders or mood disorders [12-16]. These findings support the existence of a range of cognitive impairments across different syndromes with evidence for two [14], three $[13,16]$ and four $[12,15]$ cognitive subgroups.

Furthermore, emerging evidence suggests that cluster analysis can identify phenotypes that relate more closely to specific clinical and functional trajectories than existing diagnostic categories [17]. Indeed, such approaches have highlighted poorer functioning and greater symptom severity in cognitively impaired vs. cognitively spared subgroups in schizophrenia-spectrum populations [12, 15, 18-21]. Moreover, subgroups with impaired cognition have also been associated with reductions in brain volume [22, 23] and different profiles of treatment response [24].

There is preliminary evidence for similar profiles of cognitive impairment in FEP patients, with little consensus on the number of emergent clusters [25-28]. Wenzel et al. [28] and Reser et al. [25] identified two and four cognitive subgroups in FEP patients, respectively, with high negative symptom severity and low premorbid IQ characteristic of the most cognitively impaired subgroup. Interestingly, Uren et al. [27] and Sauvé et al. [26] both obtained a three-cluster solution and found that $28 \%$ and $54 \%$ of FEP participants, respectively, aggregated with HCs in the cognitively spared subgroup, supporting the existence of an FEP subgroup with intact cognitive functioning. According to Uren et al. [27], cluster membership was associated with symptom severity and functioning from baseline to 6 months, highlighting the potential utility of cognitive clustering for prognosis and early intervention.

To our knowledge, only one study has used cluster analysis to examine cognitive profiles in CHR-P participants. Velthorst et al. [29] derived four distinct cognitive subgroups, whereby $44 \%$ of CHR-P participants were significantly or mildly impaired and 56\% displayed average or above average cognitive scores. In addition, cognitive subgroups yielded prognostic information with cluster membership predicting conversion to psychosis over a 30-month follow-up period. However, this study did not examine the predictive utility of cognitive subgroups in relation to global functioning or symptom persistence and did not include any measures of social cognition. Furthermore, it is unclear whether these findings from help-seeking CHR-P participants would generalise to more representative samples recruited outside clinical pathways.

To address these important questions, we sought to identify cognitive clusters in a sample of CHR-P and FEP participants, primarily recruited from the community, alongside individuals who did not fulfil CHR-P criteria but were characterised by affective and substance use disorders (CHRNs) and HCs. Specifically, we performed cluster analysis on principal components derived from both neurocognitive and social cognitive measures. We then examined the distribution of diagnostic groups across clusters and investigated whether cognitive subgroups were associated with clinical and functional variables at baseline and follow-up in the CHR-P group. Given previous findings in CHR-P and FEP samples [25-27, 29], we hypothesised the existence of at least three distinct cognitive profiles. In addition, we expected CHR-P individuals with pronounced cognitive deficits to exhibit the poorest functioning and greatest symptom severity at baseline and follow-up as well as cluster membership to predict clinical and functional outcomes in the CHR-P group.

\section{Methods}

\section{Participants}

Participants were recruited through the ongoing Youth Mental Health Risk and Resilience (YouR) study [30] which seeks to identify neurobiological and psychological mechanisms and predictors of psychosis risk. CHR-P participants from the general population were recruited through an online-screening approach (www.your-study.org.uk) [31]. FEP and CHR-N participants were also recruited using this method while $\mathrm{HCs}$ were obtained from a volunteer database. A smaller number of CHR-P and FEP individuals were also recruited via referrals from clinical services in NHS Greater 
Glasgow and Clyde and NHS Lothian as well as student counselling services. Ethical approval was obtained from the West of Scotland Research Ethics Service and the University of Glasgow. All participants provided written informed consent.

Baseline data were available for 146 CHR-P participants, 15 participants with first-episode psychosis (FEP), 47 participants who did not fulfil CHR-P criteria (CHR$\mathrm{Ns}$ ) and 53 healthy controls (HCs). Unlike HCs, CHR-N participants met criteria for mood and anxiety disorders as well as substance use. Thus, the inclusion of the CHR-N group allowed us to potentially disentangle the impact of psychiatric comorbidity from the CHR-P state since mood and anxiety disorders are common in this population [32]. Referred participants comprised $11.0 \%$ of the CHR-P sample and $46.7 \%$ of the FEP sample. One hundred and twenty-two CHR-P participants (83.6\%) also completed a follow-up session 6- and/or 12-months later.

Previous publications by our group have reported baseline demographic, clinical, functional and cognitive data from similar or smaller samples [31, 33-35].

\section{Baseline assessments}

To establish CHR-P criteria, participants received the positive scale of the Comprehensive Assessment of At-Risk Mental States (CAARMS) [6] and the Cognitive Disturbances (COGDIS) and Cognitive-Perceptive Basic Symptoms (COPER) items of the Schizophrenia Proneness Instrument, Adult version (SPI-A) [36].

Participants were recruited into the CHR-P group if they met one or both SPI-A criteria (i.e. COGDIS, COPER) and/ or at least one of the following CAARMS criteria: APS, genetic risk and functional deterioration (GRFD), brief limited intermittent psychotic symptoms (BLIPS). FEP criteria were established using the Structured Clinical Interview for DSM-IV (SCID) [37] and the Positive and Negative Syndrome Scale (PANSS) [38].

Cognitive assessments consisted of the Brief Assessment of Cognition in Schizophrenia (BACS) [39] and three tasks from the Penn Computerized Neurocognitive Battery (CNB) [40]: the Continuous Performance Test, the N-Back Test and the Emotion Recognition Task which provide measures of accuracy and response time (RT) for attention, working memory and emotion recognition respectively (Supplementary Table 1). Furthermore, with the exception of the FEP group, all participants were assessed with the Mini-International Neuropsychiatric Interview (MINI) [41], Global Assessment of Functioning (GAF) scale from the DSM-IVTR, Global Functioning: Social (GF: Social) and Role (GF: Role) scales [42], Premorbid Adjustment Scale (PAS) [43] and National Adult Reading Test (NART) [44].

\section{Clinical and functional outcome}

CHR-P participants were invited for follow-up interviews at 6- and 12-months. These involved the positive scale of the CAARMS as well as the GAF, GF: Social and GF: Role scales. Based on the most recent GAF score, CHR-P participants were divided into good functional outcome $(\mathrm{GAF} \geq 65)$ and poor functional outcome $(\mathrm{GAF}<65)$ groups, in line with previous research $[45,46]$. CAARMS persistence was operationalised as meeting APS criteria at both baseline and the latest follow-up assessment. Transitions to psychosis, recorded over a 36-month follow-up period, were also defined according to CAARMS criteria and subsequently followed up with the Structured Clinical Interview for DSMIV (SCID) [37] to establish the specific psychosis diagnoses.

\section{Statistical analysis}

Data were analysed using $\mathrm{R}$ version 4.0.1 [47] with statistical significance set at $p<0.05$ (two-tailed). Overall, $0.48 \%$ of the data (52 of 10,904 values) were missing and imputed by Bayesian imputation.

In line with Keefe et al. [48], BACS raw scores for each cognitive domain were converted into standardized $\mathrm{z}$-scores using the means and standard deviations (SDs) of sex-specific HCs. For consistency, CNB raw accuracy and RT scores were calculated in the same way, albeit without correction for sex. RT z-scores were multiplied by -1 , to produce speed values where, as for accuracy, higher scores reflect better performance. CNB efficiency scores were then generated for each domain by taking the arithmetic mean of the accuracy and RT z-scores. Outliers beyond $\pm 5.0 \mathrm{z}$-scores were curtailed to values of +5.0 or -5.0 . NART-derived estimates of premorbid full-scale IQ were obtained using a recently re-standardised calculation [49]. CAARMS severity was calculated by multiplying the global score by the frequency score for each domain and summing these products [50] while SPI-A severity was calculated by summing the frequency scores for each basic symptom.

In the first step, a principal component analysis (PCA) was conducted on 20 cognitive tests with oblique (oblimin) rotation, so as to allow for possible correlations between the factors, using the psych [51] and GPArotation [52] packages. Data suitability for PCA was assessed with the Kaiser-Meyer-Olkin (KMO) measure of sampling adequacy $[53,54]$ and Bartlett's test of sphericity [55]. To determine the appropriate number of principle components to extract, we used the Kaiser criterion of eigenvalues $>1$ [56] as well as scree plot inspection [57]. Cronbach's $\alpha$ was used to determine the internal consistency of data.

In the second step, we evaluated the clustering tendency of our data as well as the optimal clustering approach. Clustering tendency of the resulting component scores was 
assessed using the Hopkins $(\mathrm{H})$ statistic via the clustertend package [58]. A value close to 1 indicates uniformly distributed data while highly clustered data yields a value close to 0 . To identify the optimal clustering algorithm and number of clusters, we used the clValid package [59] which simultaneously compares the different clustering solutions in terms of validation measures. We tested for the presence of two to six clusters, implementing three clustering methods: (1) k-means, (2) partitioning around medoids (PAM) and (3) agglomerative hierarchical clustering. Internal validation measures were calculated as connectivity, silhouette width and Dunn index. Stability validation measures comprised the average proportion of non-overlap (APN), the average distance (AD), the average distance between means (ADM) and the figure of merit (FOM). Whereas internal validation measures evaluate the connectedness, compactness and separation of the different clusters, stability validation measures assess the consistency of a clustering result by comparing it with the clusters obtained after removing each column, one at a time. In general, smaller values reflect better performance, with the exception of silhouette width and Dunn index where larger values are preferable. This information was used to inform the third step whereby data-driven agglomerative hierarchical clustering was applied to the component scores via the stats package [47], using Ward's method and squared Euclidean distance, to produce two clusters. Cross-validated linear discriminant analysis, using the 20 original standardised cognitive scores as independent variables, was performed with the caret package [60] to evaluate the classification accuracy of the final clustering solution.

For the CHR-P group, the resulting clusters were compared on demographic, functional, clinical and cognitive characteristics using Welch's $t$ tests, Mann-Whitney $U$ tests, Pearson's chi-squared tests and Fisher's exact tests. We also conducted a series of hierarchical multiple linear regression analyses to examine effects of cluster membership on cognitive domains and functional variables after controlling for the potential effects of clinical (CAARMS and SPI-A severity) and demographic (age, sex, education) variables to examine the possibility that differences by cluster were better accounted for by overall symptom severity or demographic characteristics. In these models, clinical and demographic variables were entered in step 1 and cluster membership was entered in step 2. Binary logistic regression analyses were also employed to determine whether cluster membership could predict clinical and functional outcomes. The overall variance explained was measured by the Nagelkerke pseudo $R^{2}$ statistic $\left(R^{2} N\right)$ while diagnostic accuracy was determined using the area under the receiver operating characteristic (ROC) curve (AUC).

\section{Results}

\section{Demographic data}

CHR-P individuals had significantly fewer years of education, greater symptom severity, higher likelihood of comorbid mood and anxiety disorders and poorer functioning compared to CHR-N and HC participants (Table 1). Relative to the total sample, CHR-P individuals were also significantly younger while FEP patients displayed significantly higher CAARMS severity, antipsychotic and anxiolytic medication use as well as poorer global functioning. Among the CHR-P group, 43 (29.5\%) met CAARMS criteria, 34 (23.3\%) met SPI-A criteria and 69 (47.3\%) met both. Moreover, the FEP group comprised participants with SCID DSM-IV schizophrenia $(n=10$; $66.7 \%)$, psychotic disorder not otherwise specified ( $n=3$; $20.0 \%)$, schizoaffective disorder $(n=1 ; 6.7 \%)$ and schizophreniform disorder $(n=1 ; 6.7 \%)$.

\section{Principal component analysis}

The KMO measure verified the sampling adequacy for the PCA $(\mathrm{KMO}=0.70)$ with all values for individual items $\geq 0.52$, which is above the acceptable limit of 0.50 . Bartlett's test of sphericity, $\chi^{2}(190)=3795.385, p<0.001$, indicated that correlations between items were sufficiently large for PCA. Five principal components were extracted and, in combination, explained $68 \%$ of the variance in cognitive performance (Fig. 1; Supplementary Tables 2 and 3). These were labelled verbal fluency $(\alpha=0.89)$, emotion recognition ( $\alpha=0.82)$, attention ( $\alpha=0.93)$, working memory $(\alpha=0.88)$ and general cognitive function $(\alpha=0.68)$.

\section{Agglomerative hierarchical cluster analysis}

The resulting dataset contained statistically meaningful clusters $(H=0.24)$. All internal validation measures and two out of four stability validation criteria favoured agglomerative hierarchical clustering with two clusters. The dendrogram was cut to produce two clusters and subjects were assigned cluster membership accordingly (Supplementary Fig. 1). Cluster 1 comprised 111 (42.5\%) cognitively impaired participants while cluster 2 comprised 150 (57.5\%) cognitively spared participants. Linear discriminant analysis with tenfold repeated (100 times) cross-validation, using the 20 original standardised cognitive scores as independent variables, confirmed that we were able to predict the cluster membership of new cases with a mean accuracy of $88.8 \%$.

Cluster 1 comprised $93.3 \%(n=14)$ of FEP individuals and $45.9 \%(n=67)$ of CHR-P participants (Fig. 2). In 
Table 1 Demographic, clinical and functional characteristics of the total sample $(N=261)$ at baseline

\begin{tabular}{|c|c|c|c|c|c|c|}
\hline & $\begin{array}{l}\text { CHR-P (1) } \\
(N=146)\end{array}$ & $\begin{array}{l}\text { FEP (2) } \\
(N=15)\end{array}$ & $\begin{array}{l}\text { CHR-N (3) } \\
(N=47)\end{array}$ & $\begin{array}{l}\mathrm{HC}(4) \\
(N=53)\end{array}$ & Effect size $^{\mathrm{a}}$ & Post hoc test ${ }^{b}$ \\
\hline Age (years), mean (SD) & $21.47(4.22)$ & $24.40(4.37)$ & $22.94(4.80)$ & $22.42(3.36)$ & $0.003 \eta_{p}^{2}=0.051$ & $2,3,4>1$ \\
\hline Sex, female $n(\%)$ & $104(71.2)$ & $7(46.7)$ & $30(63.8)$ & $36(67.9)$ & $0.241 \quad V=0.127$ & \\
\hline Education (years), mean (SD) & $15.12(3.10)$ & $15.25(2.84)$ & $16.45(3.44)$ & $16.47(2.85)$ & $0.010 \eta_{p}^{2}=0.043$ & $3,4>1$ \\
\hline CAARMS severity, median (range) & $28(0-74)$ & $79(38-122)$ & $6(0-24)$ & $0(0-12)$ & $<0.001 \eta_{p}^{2}=0.404$ & $2>1>3>4$ \\
\hline SPI-A severity, median (range) & $7(0-74)$ & $15(0-109)$ & $0(0-7)$ & $0(0-2)$ & $<0.001 \eta_{p}^{2}=0.336$ & $1,2>3,4$ \\
\hline GAF, median (range) & $58(21-95)$ & $41(18-79)$ & $70(43-94)$ & $88(67-97)$ & $<0.001 \eta_{p}^{2}=0.343$ & $4>3>1>2$ \\
\hline $\begin{array}{l}\text { Social functioning (current), median } \\
\text { (range) }\end{array}$ & $8(3-10)$ & & $8(6-9)$ & $9(8-10)$ & $<0.001 \eta_{p}^{2}=0.229$ & $4>3>1$ \\
\hline Role functioning (current), median (range) & $8(3-9)$ & & $8(5-9)$ & $9(5-9)$ & $<0.001 \eta_{p}^{2}=0.199$ & $4>3>1$ \\
\hline PAS average, median (range) & $1.28(0-3.43)$ & & $0.86(0-3.86)$ & $0.43(0-1.64)$ & $<0.001 \eta_{p}^{2}=0.189$ & $1>3>4$ \\
\hline \multicolumn{7}{|l|}{ Comorbidity, $n(\%)$} \\
\hline Anxiety disorder & $104(71.2)$ & & $22(46.8)$ & $0(0)$ & $<0.001 \quad V=0.568$ & $1>3>4$ \\
\hline Mood disorder & $97(66.4)$ & & $14(29.8)$ & $0(0)$ & $<0.001 \quad V=0.552$ & $1>3>4$ \\
\hline Alcohol abuse/dependence & $46(31.5)$ & & $11(23.4)$ & $0(0)$ & $<0.001 \quad V=0.297$ & $1,3>4$ \\
\hline Substance abuse/dependence & $24(16.4)$ & & $3(6.4)$ & $0(0)$ & $0.002 \quad V=0.221$ & $1>4$ \\
\hline Eating disorder & $13(8.9)$ & & $1(2.1)$ & $0(0)$ & $0.023 \quad V=0.170$ & $1>4$ \\
\hline \multicolumn{7}{|l|}{ Psychological treatment, $n(\%)$} \\
\hline Current & $25(17.1)$ & $5(33.3)$ & $5(10.6)$ & $0(0)$ & $0.002 \quad V=0.243$ & $2>3,4$ and $1>4$ \\
\hline Past & $66(45.2)$ & $5(33.3)$ & $15(31.9)$ & $3(5.7)$ & $<0.001 \quad V=0.323$ & $1,2,3>4$ \\
\hline \multicolumn{7}{|l|}{ Medication, $n(\%)$} \\
\hline Antidepressants & $53(36.3)$ & $9(60.0)$ & $13(27.7)$ & $0(0)$ & $<0.001 \quad V=0.354$ & $2>3,4$ and $1>4$ \\
\hline Mood stabilisers & $4(2.7)$ & $0(0)$ & $0(0)$ & $0(0)$ & $0.592 \quad V=0.111$ & \\
\hline Antipsychotics & $4(2.7)$ & $7(46.7)$ & $0(0)$ & $0(0)$ & $<0.001 \quad V=0.526$ & $2>1,3,4$ \\
\hline Anxiolytics & $10(6.8)$ & $5(33.3)$ & $1(2.1)$ & $0(0)$ & $<0.001 \quad V=0.304$ & $2>1,3,4$ \\
\hline
\end{tabular}

$C H R-P$ clinical high-risk for psychosis, $F E P$ first-episode psychosis, $C H R-N$ clinical high-risk-negative, $H C$ healthy control, $C A A R M S$ Comprehensive Assessment of At-Risk Mental States, SPI-A Schizophrenia Proneness Instrument, Adult version, GAF Global Assessment of Functioning, PAS Premorbid Adjustment Scale

aEffect sizes were eta squared $\left(\eta_{p}^{2}\right)$ for Kruskal-Wallis $H$ tests (small effect $=0.01$, medium effect $=0.06$, large effect $=0.14$ ) and Cramer's V for Pearson's chi-squared or Fisher-Freeman-Halton tests ( small effect $=0.1$, medium effect $=0.3$, large effect $=0.5$ )

${ }^{\mathrm{b}} 1=$ CHR-P, $2=$ FEP, $3=$ CHR-N, $4=$ HC

addition, similar percentages of CHR-N and HC individuals were assigned to cluster 1 (CHR-N: 29.8\%; HC: $30.2 \%)$.

\section{Cluster comparisons at baseline}

CHR-P individuals in cluster 1 displayed significantly lower premorbid IQ and poorer performance across all $20 \mathrm{cog}$ nitive tests compared to those in cluster $2(p<0.01)$, with medium to large effect sizes (Supplementary Table 4), and were characterised by poorer social, role and premorbid functioning $(p<0.01)$ but not global functioning (Table 2; Fig. 3). Male CHR-P participants were also significantly more likely $(p<0.001)$ to be allocated to cluster $1(47.8 \%)$ than cluster $2(12.7 \%)$. After controlling for clinical symptoms and demographic characteristics, cluster membership remained significantly associated with premorbid IQ $(t=2.565 ; p=0.011)$, all 20 cognitive domains $(t=2.033$ to $7.166 ; p<0.05)$, social functioning $(t=2.375 ; p=0.019)$ and premorbid functioning $(t=-3.997 ; p<0.001)$, but not role functioning $(t=1.548 ; p=0.124)$. Furthermore, the proportion of CHR-P participants meeting CAARMS criteria, SPI-A criteria or both did not differ between the clusters $(p=0.667)$.

\section{Cluster comparisons and outcome prediction at follow-up}

CHR-P individuals in cluster 1 displayed significantly poorer global and social functioning at follow-up 6- and/or 12 -months later compared to those in cluster $2(p<0.05)$. Within the CHR-P group, poor functional outcome was also significantly more likely $(p=0.007)$ in cluster $1(71.9 \%)$ compared to cluster $2(47.7 \%)$.

In a binary logistic regression analysis, cluster membership explained $8.0 \%$ of the variance in functional outcome $(p=0.007$, AUC $=0.625$, sensitivity $=56.9 \%$ 


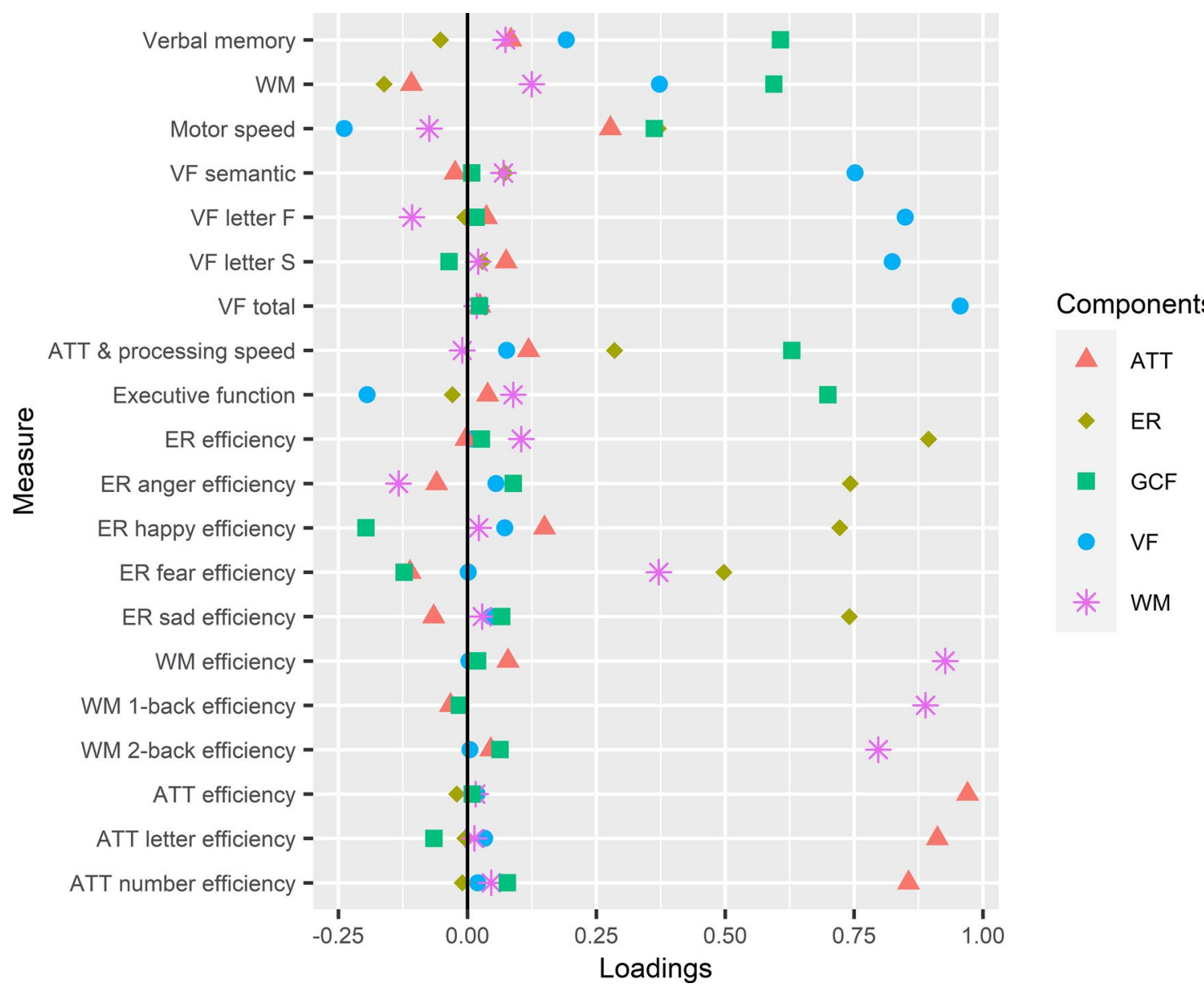

Fig. 1 Component loading plot for the total sample $(N=261)$. ATT attention, $E R$ emotion recognition, $G C F$ general cognitive function, $V F$ verbal fluency, $W M$ working memory

and specificity $=68.0 \%$ ). Based on the odds ratio, poor functional outcome was 2.81 times higher if participants were assigned to cluster 1 rather than cluster 2. This association remained significant after adjusting for GAF score at baseline (adjusted odds ratio $=2.52, p=0.030$ ). In contrast, cluster membership could not predict clinical outcomes in terms of CAARMS persistence $(p=0.768)$ or transition to psychosis $(p=0.170)$.

\section{Additional analyses}

The PCA and cluster analysis were repeated following the exclusion of the small sample of FEP participants to verify the stability and interpretability of our results. Overall, results remained unchanged, albeit with slightly smaller effect sizes (see Supplementary Results and Supplementary Figs. 2-5).

\section{Discussion}

Using a data-driven hierarchical clustering approach in conjunction with PCA, we identified a two-cluster solution, comprising a cognitively spared and cognitively impaired subgroup, in a sample consisting of CHR-P and FEP participants as well as CHR-N participants and HCs. While the majority of FEP individuals were assigned to the cognitively impaired cluster, CHR-P individuals were almost equally distributed. At both baseline and followup, CHR-P individuals classified as cognitively impaired displayed significantly poorer functioning than their cognitively spared counterparts with cluster membership able to predict functional but not clinical outcome. 


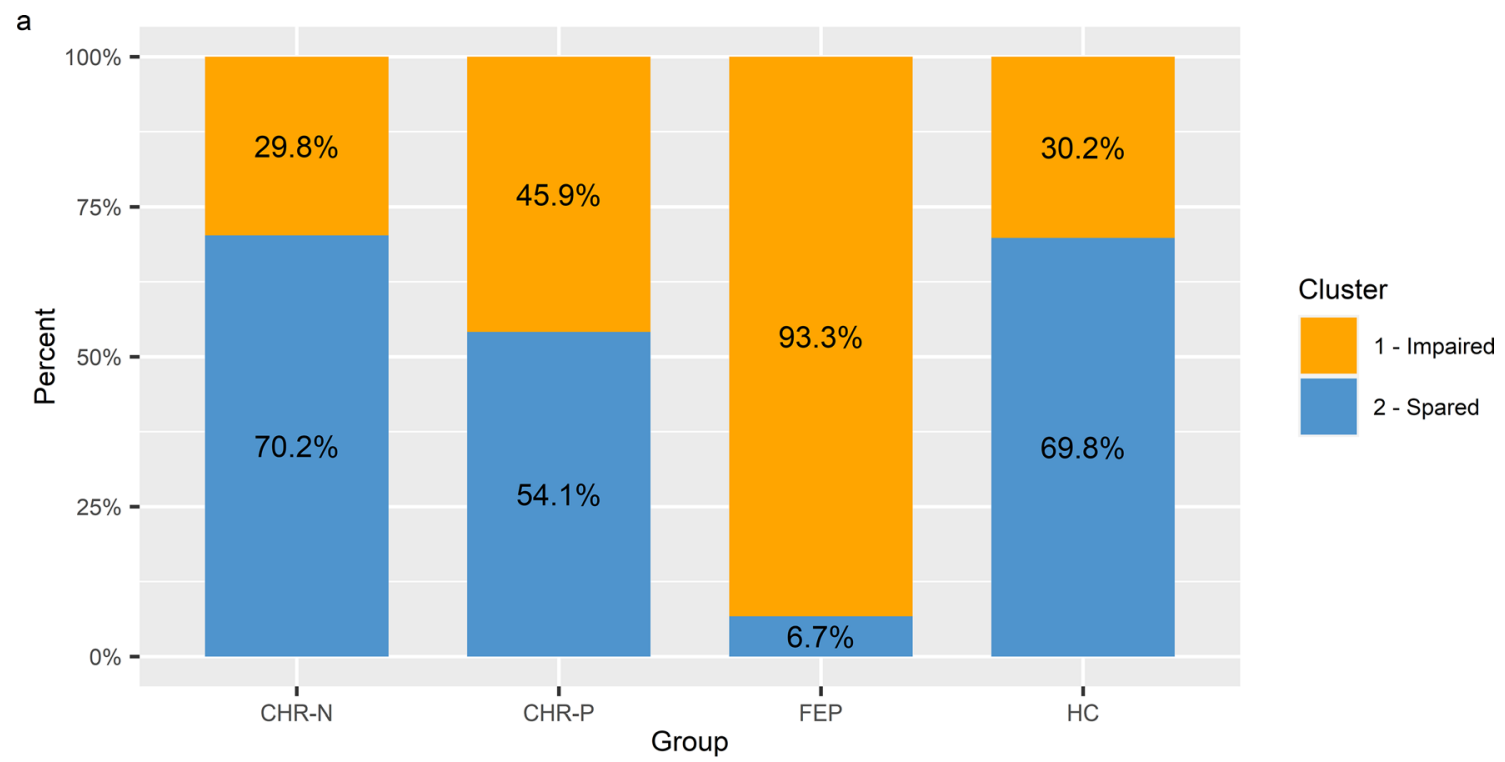

b
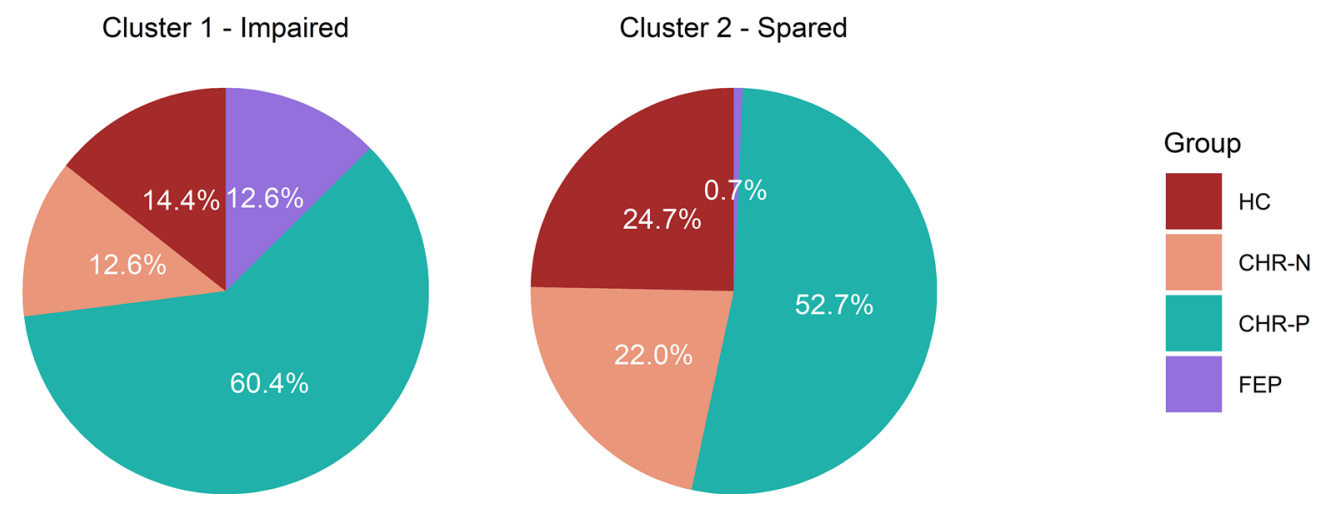

Fig. 2 The distribution of a clusters within each diagnostic group and $\mathbf{b}$ diagnostic groups within each cluster for the total sample $(N=261)$. $C H R-P$ clinical high-risk for psychosis, $F E P$ first-episode psychosis, $C H R-N$ clinical high-risk-negative; $H C$ healthy control

\section{Hierarchical clustering on principal components}

In the present study, PCA was applied prior to clustering to reduce data dimensionality, thereby reducing information redundancy and maximising explanatory variance [61]. Verbal fluency, emotion recognition, attention, working memory and general cognitive function were the five principal components that explained $68 \%$ of the variance in cognitive performance across the entire sample. Interestingly, Lam et al. [62] observed a similar cognitive component structure in both CHR-P and HC samples, indicating that our components constitute reproducible dimensions of cognitive performance.

The emergence of a two-cluster solution is in agreement with previous studies involving schizophrenia-spectrum disorders [14, 20, 28, 63, 64]. However, three- or four-cluster solutions are more typically reported in mixed samples of
FEP and HC participants [26, 27]. Furthermore, the only study to investigate cognitive subgroups in CHR-P participants obtained a four-cluster solution [29]. It is possible that our two-cluster solution partially reflects the novel combination of FEP and CHR-P participants as well as the application of basic symptom criteria to recruit CHR-P individuals. Nevertheless, this solution has resulted from replicable cognitive components [62], supporting the validity of our findings.

Finally, it is important to note that the majority of CHR-P participants in the current study were recruited from the community and not through dedicated clinical pathways. Community-recruitment strategies represent an important aspect of early detection and intervention $[65,66]$. Indeed, there may be a substantial number of young people at CHR-P in the community who are not seen by specialised early detection services [65]. Therefore, community-recruitment strategies are 
Table 2 Demographic, clinical and functional characteristics of the CHR-P group by cognitive cluster at baseline $(N=146)$ and follow-up $(N=122)$

\begin{tabular}{|c|c|c|c|c|}
\hline Baseline & $\begin{array}{l}\text { Cluster } 1 \\
\text { Impaired }(N=67)\end{array}$ & $\begin{array}{l}\text { Cluster } 2 \\
\text { Spared }(N=79)\end{array}$ & $p$ & Effect size $^{\mathrm{a}}$ \\
\hline Age (years), mean (SD) & $21.36(4.63)$ & $21.56(3.86)$ & 0.288 & $r=0.088$ \\
\hline Sex, female $n(\%)$ & $35(52.2)$ & $69(87.3)$ & $<0.001$ & $\phi=0.386$ \\
\hline Education (years), mean (SD) & $14.96(3.43)$ & $15.25(2.80)$ & 0.421 & $r=0.067$ \\
\hline CAARMS severity, median (range) & $29(0-74)$ & $28(0-72)$ & 0.212 & $r=0.103$ \\
\hline SPI-A severity, median (range) & $6(0-61)$ & $7(0-74)$ & 0.883 & $r=0.012$ \\
\hline GAF, median (range) & $55(21-87)$ & $60(21-95)$ & 0.094 & $r=0.139$ \\
\hline Social functioning (current), median (range) & $7(3-10)$ & $8(3-10)$ & $<0.001$ & $r=0.296$ \\
\hline Role functioning (current), median (range) & $7(4-9)$ & $8(3-9)$ & 0.002 & $r=0.255$ \\
\hline PAS average, median (range) & $1.36(0-3.43)$ & $0.86(0-2.57)$ & $<0.001$ & $r=0.405$ \\
\hline \multicolumn{5}{|l|}{ Comorbidity, $n(\%)$} \\
\hline Anxiety disorder & $49(73.1)$ & $55(69.6)$ & 0.640 & $\phi=0.039$ \\
\hline Mood disorder & $50(74.6)$ & $47(59.5)$ & 0.054 & $\phi=0.160$ \\
\hline Alcohol abuse/dependence & $18(26.9)$ & $28(35.4)$ & 0.266 & $\phi=0.092$ \\
\hline Substance abuse/dependence & $11(16.4)$ & $13(16.5)$ & 0.995 & $\phi=0.001$ \\
\hline Eating disorder & $4(6.0)$ & $9(11.4)$ & 0.252 & $\phi=0.095$ \\
\hline \multicolumn{5}{|l|}{ Psychological treatment, $n(\%)$} \\
\hline Current & $15(22.4)$ & $10(12.7)$ & 0.120 & $\phi=0.129$ \\
\hline Past & $27(40.3)$ & $39(49.4)$ & 0.273 & $\phi=0.091$ \\
\hline \multicolumn{5}{|l|}{ Medication, $n(\%)$} \\
\hline Antidepressants & $25(37.3)$ & $28(35.4)$ & 0.815 & $\phi=0.019$ \\
\hline Mood stabilisers & $2(3.0)$ & $2(2.5)$ & 10.000 & $\phi=0.014$ \\
\hline Antipsychotics & $3(4.5)$ & $1(1.3)$ & 0.333 & $\phi=0.098$ \\
\hline Anxiolytics & $4(6.0)$ & $6(7.6)$ & 0.754 & $\phi=0.032$ \\
\hline \multirow[t]{2}{*}{ Follow-Up } & Cluster 1 & Cluster 2 & $p$ & Effect size $^{\mathrm{a}}$ \\
\hline & Impaired $(N=57)$ & Spared $(N=65)$ & & \\
\hline GAF, median (range) & $52(21-88)$ & $68(33-88)$ & 0.012 & $r=0.227$ \\
\hline Poor functional outcome, $n(\%)$ & $41(71.9)$ & $31(47.7)$ & 0.007 & $\phi=0.246$ \\
\hline Social functioning (current), median (range) & $8(2-10)$ & $8(4-9)$ & 0.021 & $r=0.209$ \\
\hline Role functioning (current), median (range) & $8(4-9)$ & $8(5-9)$ & 0.139 & $r=0.134$ \\
\hline CAARMS severity, median (range) & $15(0-71)$ & $12(0-82)$ & 0.886 & $r=0.013$ \\
\hline CAARMS persistence, $n(\%)$ & $17(29.8)$ & $21(32.3)$ & 0.768 & $\phi=0.027$ \\
\hline Transitions $^{\mathrm{b}}, n(\%)$ & $9(15.8)$ & $5(7.7)$ & 0.162 & $\phi=0.127$ \\
\hline
\end{tabular}

CHR-P clinical high-risk for psychosis, CAARMS Comprehensive Assessment of At-Risk Mental State, SPI-A Schizophrenia Proneness Instrument, Adult version, GAF Global Assessment of Functioning, $P A S$ premorbid adjustment scale

${ }^{a}$ Effect sizes were Rosenthal's $\mathrm{r}$ for Mann-Whitney $U$ tests and Phi $(\phi)$ for Pearson's chi-squared or Fisher's exact tests (small effect $=0.1$, medium effect $=0.3$, large effect $=0.5$ )

${ }^{\mathrm{b}} 19$ non-transitioned CHR-P individuals have yet to reach the 3-year follow-up

particularly advantageous in their ability to detect more representative samples, ensuring that findings can be generalised to the entire population of individuals at CHR-P.

\section{Characterising within-group cognitive heterogeneity}

In line with Velthorst et al. [29], our CHR-P group exhibited substantial cognitive heterogeneity, with $45.9 \%$ of individuals assigned to the cognitively impaired subgroup. On the other hand, cognitive heterogeneity was less apparent in our FEP group, contrasting with previous findings in larger 

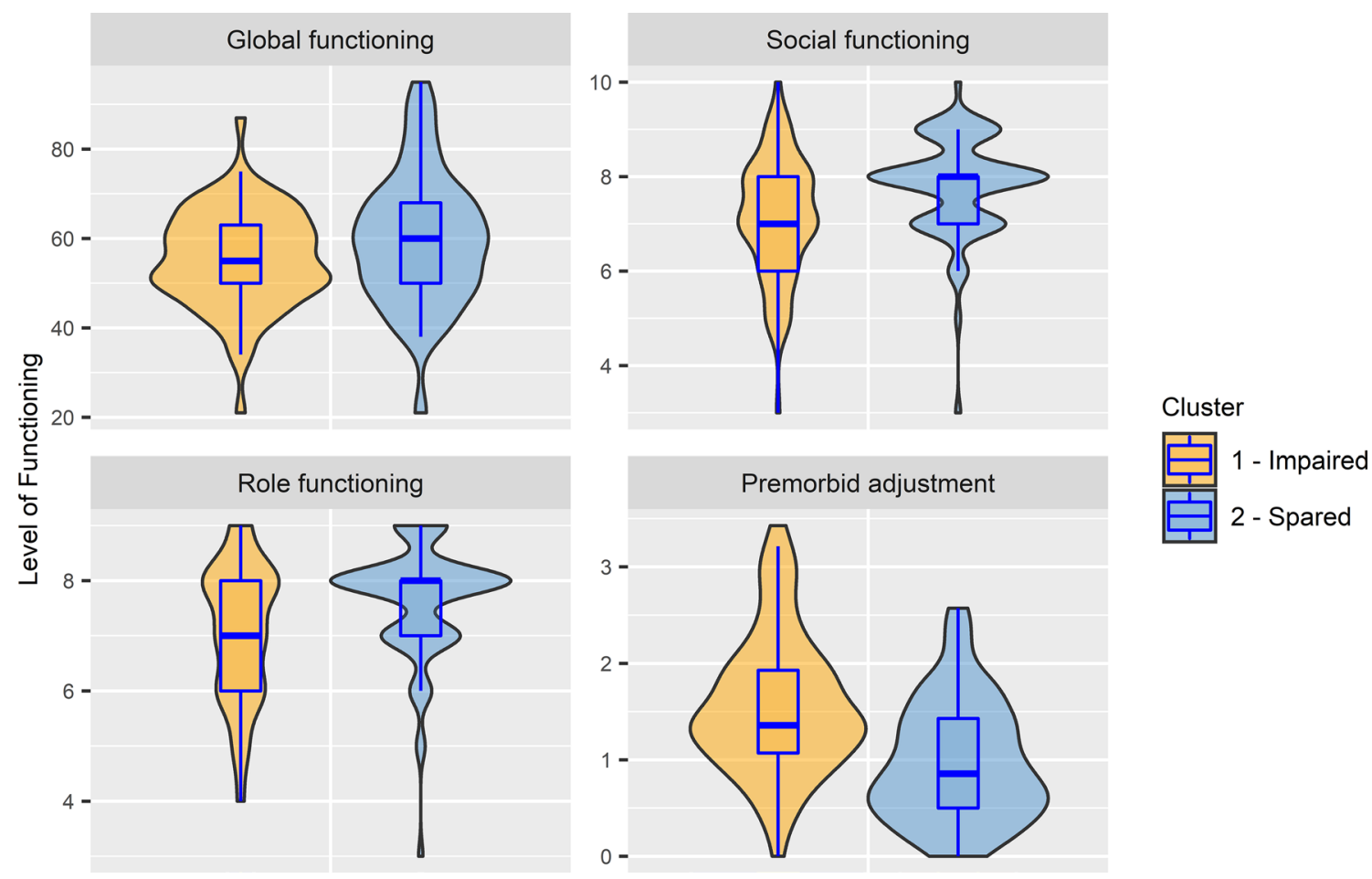

Fig. 3 Level of functioning across cognitive clusters for the CHR-P group $(N=146)$

samples [26, 27]. Approximately 16\% fewer CHR-N participants were classified as cognitively impaired relative to CHR-P participants, indicating that cognitive impairment is somewhat more prevalent in the CHR-P state. Interestingly, a considerable proportion of HCs (30.2\%) were also allocated to the cognitively impaired subgroup, supporting previous findings [26]. Overall, these results support the notion of a cognitive continuum [12, 16, 67], at least among CHR-P, CHR-N and HC populations.

\section{Cluster comparisons in the CHR-P group}

Cognitively impaired CHR-P individuals displayed significantly poorer performance across all domains with large effect sizes for verbal memory, verbal fluency and attention and processing speed. Indeed, cognitive scores fell mostly within 0.5-1.0 SDs below HC data for cognitively impaired participants. Deficits in facial emotion recognition were also significantly greater in cognitively impaired individuals with medium effect sizes, indicating that cluster membership was driven by the degree of impairment across both neurocognitive and social cognitive domains.

Within the CHR-P group, cognitively impaired individuals had significantly poorer functioning than cognitively spared individuals. While role functioning and global functioning were significantly reduced at baseline and follow-up, respectively, social functioning was impaired at both time points, in line with previous findings [29]. Lower levels of premorbid functioning and premorbid IQ were also observed in the cognitively impaired vs. cognitively spared subgroup, consistent with previous studies across the psychosis spectrum $[15,18,25,27]$. These findings, in addition to the larger number of male participants in our cognitively impaired subgroup, may support the existence of a neurodevelopmental contribution towards pronounced cognitive impairments in CHR-P participants [68], in line with previous results in psychosis patients [69].

In contrast, positive symptom severity did not significantly differ between cognitive subgroups. Cluster analyses have produced mixed findings, reporting either no significant differences across cognitive subgroups in the schizophreniaspectrum $[18,25,28,63]$ or greater positive symptom severity in the most cognitively impaired cluster [12, 15, 26, 27]. Furthermore, the proportion of CHR-P participants meeting CAARMS criteria, SPI-A criteria or both did not differ between the cognitive subgroups, contrasting with previous reports of lesser cognitive deficits in individuals meeting basic symptom, as opposed to UHR, criteria [70].

\section{Outcome prediction in the CHR-P group}

Importantly, we were also able to predict functional outcome from cluster membership, with cognitively impaired CHR-P individuals significantly more likely to experience 
poor functional outcome at follow-up. Conversely, cluster membership was unable to predict clinical outcomes in terms of APS persistence or transition to psychosis. This contrasts with Velthorst et al. [29] whereby impaired cognition in CHR-P individuals predicted transition to psychosis. Nevertheless, our findings suggest that early interventions targeting cognition, such as cognitive remediation, should be tailored towards cognitively impaired CHR-P participants to alleviate cognitive deficits and consequently improve functional outcome [71].

\section{Limitations}

Certain limitations should be considered. First, the sample size of FEP participants was small, limiting our ability to accurately characterise cognitive heterogeneity in this group. Furthermore, negative symptoms were not assessed in the current study while cognition was only assessed at baseline. Therefore, we were unable to ascertain the full impact of clinical symptomatology on cluster assignment as well as the stability of cognitive subgroups over time. Finally, cluster membership explained only $8.0 \%$ of the variance in functional outcome. This could, in part, be explained by our measure of functioning. For example, the GAF scale confounds functioning with symptom severity, the latter being unrelated to functioning in the current study. Nevertheless, this measure was chosen over social and role functioning scales as these scores were mostly limited in range.

\section{Conclusions}

We employed cluster analysis to investigate cognitive subgroups in CHR-P participants using a community-recruitment approach, social cognitive measures and functional outcome prediction. We identified two discrete cognitive subgroups and found support for considerable cognitive heterogeneity within the CHR-P group. Cognitively impaired and cognitively spared CHR-P individuals could be distinguished on measures of functioning at baseline and followup, with cluster membership able to predict functional outcome. These findings emphasise the key role cognition plays in functioning and suggest that cluster assignment is driven by cognitive performance, rather than clinical symptoms. In addition, the current findings may support the role of cognitive enhancement therapies, such as cognitive remediation, in CHR-P individuals with impaired cognition. Indeed, datadriven approaches such as cluster analysis could effectively stratify heterogenous clinical populations along dimensions of interest and thus represent an important step towards personalised psychiatry. Future research should attempt to replicate these findings in larger samples, over longer follow-up periods and also investigate whether these cognitive subgroups are differentially associated with neurobiological measures, such as measures of cortical thickness and volume as well as electrophysiological parameters.

Supplementary Information The online version contains supplementary material available at https://doi.org/10.1007/s00406-021-01315-2.

Acknowledgements We acknowledge the support of the Scottish Mental Health Research Network (http://www.smhrn.org.uk), now called the NHS Research Scotland Mental Health Network (NRS MHN: http://www.nhsresearchscotland.org.uk/research-areas/mental-health), for providing assistance with participant recruitment, interviews and cognitive assessments. We would like to thank both the participants and patients who took part in the study and the research assistants of the YouR-study for supporting the recruitment and assessment of CHR-P participants.

Funding This study was supported by project MR/L011689/1 from the Medical Research Council (MRC).

Data and code availability Available from the corresponding author on reasonable request.

\section{Declarations}

Conflict of interest Prof. Uhlhaas has received research support from Lilly and Lundbeck outside the submitted work.

Ethical approval The authors assert that all procedures contributing to this work comply with the ethical standards of the relevant national and institutional committees on human experimentation and with the Helsinki Declaration of 1964 and its later amendments. Ethical approval was obtained from the West of Scotland Research Ethics Service and the University of Glasgow.

Consent to participate All participants provided written informed consent.

Open Access This article is licensed under a Creative Commons Attribution 4.0 International License, which permits use, sharing, adaptation, distribution and reproduction in any medium or format, as long as you give appropriate credit to the original author(s) and the source, provide a link to the Creative Commons licence, and indicate if changes were made. The images or other third party material in this article are included in the article's Creative Commons licence, unless indicated otherwise in a credit line to the material. If material is not included in the article's Creative Commons licence and your intended use is not permitted by statutory regulation or exceeds the permitted use, you will need to obtain permission directly from the copyright holder. To view a copy of this licence, visit http://creativecommons.org/licenses/by/4.0/.

\section{References}

1. Owen MJ, Sawa A, Mortensen PB (2016) Schizophrenia. Lancet 388:86-97. https://doi.org/10.1016/S0140-6736(15)01121-6

2. Sklar AL, Coffman BA, Salisbury DF (2020) Localization of early-stage visual processing deficits at schizophrenia spectrum illness onset using magnetoencephalography. Schizophr Bull 46:955-963. https://doi.org/10.1093/schbul/sbaa010 
3. Sheffield JM, Karcher NR, Barch DM (2018) Cognitive deficits in psychotic disorders: a lifespan perspective. Neuropsychol Rev 28:509-533. https://doi.org/10.1007/s11065-018-9388-2

4. Green MF, Horan WP, Lee J (2019) Nonsocial and social cognition in schizophrenia: current evidence and future directions. World Psychiatry 18:146-161. https://doi.org/10.1002/wps.20624

5. Schultze-Lutter F, Rahman J, Ruhrmann S et al (2015) Duration of unspecific prodromal and clinical high risk states, and early help-seeking in first-admission psychosis patients. Soc Psychiatry Psychiatr Epidemiol 50:1831-1841. https://doi.org/10.1007/ s00127-015-1093-3

6. Yung AR, Yuen HP, McGorry PD et al (2005) Mapping the onset of psychosis: the comprehensive assessment of at-risk mental states. Aust N Z J Psychiatry 39:964-971. https://doi.org/10. 1111/j.1440-1614.2005.01714.x

7. Schultze-Lutter F (2009) Subjective symptoms of schizophrenia in research and the clinic: the basic symptom concept. Schizophr Bull 35:5-8. https://doi.org/10.1093/schbul/sbn139

8. Schultze-Lutter F, Ruhrmann S, Fusar-Poli P et al (2012) Basic symptoms and the prediction of first-episode psychosis. Curr Pharm Des 18:351-357. https://doi.org/10.2174/1381612127 99316064

9. Giuliano AJ, Li H, Mesholam-Gately RI et al (2012) Neurocognition in the psychosis risk syndrome: a quantitative and qualitative review. Curr Pharm Des 18:399-415. https://doi.org/10.2174/ 138161212799316019

10. Hauser M, Zhang JP, Sheridan EM et al (2017) Neuropsychological test performance to enhance identification of subjects at clinical high risk for psychosis and be most promising for predictive algorithms for conversion to psychosis: a meta-analysis. J Clin Psychiatry 78:e28-e40

11. Seidman LJ, Shapiro DI, Stone WS et al (2016) Association of neurocognition with transition to psychosis: baseline functioning in the second phase of the north American prodrome longitudinal study. JAMA Psychiat 73:1239-1248. https://doi.org/10.1001/ jamapsychiatry.2016.2479

12. Lewandowski KE, Sperry SH, Cohen BM, Öngür D (2014) Cognitive variability in psychotic disorders: a cross-diagnostic cluster analysis. Psychol Med 44:3239-3248. https://doi.org/10.1017/ S0033291714000774

13. Lee RSC, Hermens DF, Naismith SL et al (2015) Neuropsychological and functional outcomes in recent-onset major depression, bipolar disorder and schizophrenia-spectrum disorders: a longitudinal cohort study. Transl Psychiatry 5:e555. https://doi.org/10. 1038/tp.2015.50

14. Lee J, Rizzo S, Altshuler L et al (2017) Deconstructing bipolar disorder and schizophrenia: a cross-diagnostic cluster analysis of cognitive phenotypes. J Affect Disord 209:71-79. https://doi.org/ 10.1016/j.jad.2016.11.030

15. Lewandowski KE, Baker JT, McCarthy JM et al (2018) Reproducibility of cognitive profiles in psychosis using cluster analysis. $\mathbf{J}$ Int Neuropsychol Soc 24:382-390. https://doi.org/10.1017/S1355 617717001047

16. Van Rheenen TE, Lewandowski KE, Tan EJ et al (2017) Characterizing cognitive heterogeneity on the schizophrenia-bipolar disorder spectrum. Psychol Med 47:1848-1864. https://doi.org/ 10.1017/S0033291717000307

17. Clementz BA, Sweeney JA, Hamm JP et al (2016) Identification of distinct psychosis biotypes using brain-based biomarkers. Am J Psychiatry 173:373-384. https://doi.org/10.1176/appi.ajp.2015. 14091200

18. Crouse JJ, Moustafa AA, Bogaty SER et al (2018) Parcellating cognitive heterogeneity in early psychosis-spectrum illnesses: a cluster analysis. Schizophr Res 202:91-98. https://doi.org/10. 1016/j.schres.2018.06.060
19. Vaskinn A, Haatveit B, Melle I et al (2020) Cognitive heterogeneity across schizophrenia and bipolar disorder: a cluster analysis of intellectual trajectories. J Int Neuropsychol Soc. https://doi.org/ $10.1017 / \mathrm{S} 1355617720000442$

20. Green MJ, Cairns MJ, Wu J et al (2013) Genome-wide supported variant MIR137 and severe negative symptoms predict membership of an impaired cognitive subtype of schizophrenia. Mol Psychiatry 18:774-780. https://doi.org/10.1038/mp.2012.84

21. Wells R, Swaminathan V, Sundram S et al (2015) The impact of premorbid and current intellect in schizophrenia: cognitive, symptom, and functional outcomes. npj Schizophr 1:1-8. https:// doi.org/10.1038/npjschz.2015.43

22. Van Rheenen TE, Cropley V, Zalesky A et al (2018) Widespread volumetric reductions in schizophrenia and schizoaffective patients displaying compromised cognitive abilities. Schizophr Bull 44:560-574. https://doi.org/10.1093/schbul/sbx109

23. Weinberg D, Lenroot R, Jacomb I et al (2016) Cognitive subtypes of schizophrenia characterized by differential brain volumetric reductions and cognitive decline. JAMA Psychiat 73:1251-1259. https://doi.org/10.1001/jamapsychiatry.2016.2925

24. Gilbert E, Mérette C, Jomphe V et al (2014) Cluster analysis of cognitive deficits may mark heterogeneity in schizophrenia in terms of outcome and response to treatment. Eur Arch Psychiatry Clin Neurosci 264:333-343. https://doi.org/10.1007/ s00406-013-0463-7

25. Reser MP, Allott KA, Killackey E et al (2015) Exploring cognitive heterogeneity in first-episode psychosis: What cluster analysis can reveal. Psychiatry Res 229:819-827. https://doi.org/10.1016/j. psychres.2015.07.084

26. Sauvé G, Malla A, Joober R et al (2018) Comparing cognitive clusters across first- and multiple-episode of psychosis. Psychiatry Res 269:707-718. https://doi.org/10.1016/j.psychres.2018.08.119

27. Uren J, Cotton SM, Killackey E et al (2017) Cognitive clusters in first-episode psychosis: Overlap with healthy controls and relationship to concurrent and prospective symptoms and functioning. Neuropsychology 31:787-797. https://doi.org/10.1037/neu00 00367

28. Wenzel J, Haas SS, Dwyer DB et al (2021) Cognitive subtypes in recent onset psychosis: distinct neurobiological fingerprints? Neuropsychopharmacology. https://doi.org/10.1038/ s41386-021-00963-1

29. Velthorst E, Meyer EC, Giuliano AJ et al (2019) Neurocognitive profiles in the prodrome to psychosis in NAPLS-1. Schizophr Res 204:311-319. https://doi.org/10.1016/j.schres.2018.07.038

30. Uhlhaas PJ, Gajwani R, Gross J et al (2017) The youth mental health risk and resilience study (YouR-Study). BMC Psychiatry 17:1-8. https://doi.org/10.1186/s12888-017-1206-5

31. McDonald M, Christoforidou E, Van Rijsbergen N et al (2019) Using online screening in the general population to detect participants at clinical high-risk for psychosis. Schizophr Bull 45:600 609. https://doi.org/10.1093/schbul/sby069

32. Millman ZB, Gold JM, Mittal VA, Schiffman J (2019) The critical need for help-seeking controls in clinical high-risk research. Clin Psychol Sci 7:1171-1189. https://doi.org/10.1177/2167702619 855660

33. Haining K, Karagiorgou O, Gajwani R et al (2020) Prevalence and predictors of suicidality and non-suicidal self-harm among individuals at clinical high-risk for psychosis: results from a community-recruited sample. Early Interv Psychiatry. https://doi.org/ 10.1111/eip. 13075

34. Haining K, Matrunola C, Mitchell L et al (2020) Neuropsychological deficits in participants at clinical high risk for psychosis recruited from the community: relationships to functioning and clinical symptoms. Psychol Med 50:77-85. https://doi.org/10. 1017/S0033291718003975 
35. Haining K, Brunner G, Gajwani R et al (2021) The relationship between cognitive deficits and impaired short-term functional outcome in clinical high-risk for psychosis participants: a machine learning and modelling approach. Schizophr Res 231:24-31. https://doi.org/10.1016/j.schres.2021.02.019

36. Schultze-Lutter F, Addington J, Ruhrmann S, Klosterkötter J (2007) Schizophrenia proneness instrument-adult version (SPIA). Giovanni Fioriti Editore, Rome

37. First MB, Spitzer RL, Gibbon M, Williams JBW (2002) Structured clinical interview for DSM-IV-TR axis I disorders, research version, patient edition (SCID - I/P). Biometrics Research, New York State Psychiatric Institute, New York

38. Kay SR, Fiszbein A, Opler LA (1987) The positive and negative syndrome scale (PANSS) for schizophrenia. Schizophr Bull 13:261-276. https://doi.org/10.1093/schbul/13.2.261

39. Keefe RSE, Goldberg TE, Harvey PD et al (2004) The brief assessment of cognition in schizophrenia: reliability, sensitivity, and comparison with a standard neurocognitive battery. Schizophr Res 68:283-297. https://doi.org/10.1016/j.schres.2003.09.011

40. Moore TM, Reise SP, Gur RE et al (2015) Psychometric properties of the penn computerized neurocognitive battery. Neuropsychology 29:235-246. https://doi.org/10.1037/neu0000093

41. Sheehan DV, Lecrubier Y, Sheehan K et al (1998) The mini-international neuropsychiatric interview (M.I.N.I.): the development and validation of a structured diagnostic psychiatric interview for DSM-IV and ICD-10. J Clin Psychiatry 59:22-33

42. Cornblatt BA, Auther AM, Niendam T et al (2007) Preliminary findings for two new measures of social and role functioning in the prodromal phase of schizophrenia. Schizophr Bull 33:688-702. https://doi.org/10.1093/schbul/sbm029

43. Cannon-Spoor HE, Potkin SG, Wyatt RJ (1982) Measurement of premorbid adjustment in chronic schizophrenia. Schizophr Bull 8:470-484. https://doi.org/10.1093/schbul/8.3.470

44. Nelson HE (1982) National Adult Reading Test (NART): For the assessment of premorbid intelligence in patients with dementia: Test manual. Nfer-Nelson, Windsor, UK

45. Allen P, Chaddock CA, Egerton A et al (2015) Functional outcome in people at high risk for psychosis predicted by thalamic glutamate levels and prefronto-striatal activation. Schizophr Bull 41:429-439. https://doi.org/10.1093/schbul/sbu115

46. Modinos G, Kempton MJ, Tognin S et al (2019) Association of adverse outcomes with emotion processing and its neural substrate in individuals at clinical high risk for psychosis. JAMA Psychiat 77:190-200. https://doi.org/10.1001/jamapsychiatry.2019.3501

47. R Core Team (2020) R: A language and environment for statistical computing. R Foundation for Statistical Computing, Vienna, Austria. http://www.r-project.org. Accessed 20 Dec 2020

48. Keefe RSE, Harvey PD, Goldberg TE et al (2008) Norms and standardization of the brief assessment of cognition in schizophrenia (BACS). Schizophr Res 102:108-115. https://doi.org/10. 1016/j.schres.2008.03.024

49. Bright P, Hale E, Gooch VJ et al (2018) the national adult reading test: restandardisation against the Wechsler adult intelligence scale-fourth edition. Neuropsychol Rehabil 28:1019-1027. https:// doi.org/10.1080/09602011.2016.1231121

50. Morrison AP, French P, Stewart SLK et al (2012) Early detection and intervention evaluation for people at risk of psychosis: multisite randomised controlled trial. BMJ 344:1-14. https://doi.org/ 10.1136/bmj.e2233

51. Revelle W (2020) psych: Procedures for psychological, psychometric, and personality research. Northwestern University, Evanston, Illinois. R package version 2.0.9. https://cran.r-project.org/ package $=$ psych. Accessed 09 Feb 2021

52. Bernaards CA, Jennrich RI (2005) Gradient projection algorithms and software for arbitrary rotation criteria in factor analysis. Educ
Psychol Meas 65:676-696. https://doi.org/10.1177/0013164404 272507

53. Kaiser HF (1974) An index of factorial simplicity. Psychometrika 39:31-36. https://doi.org/10.1007/BF02291575

54. Kaiser HF (1970) A second generation little jiffy. Psychometrika 35:401-415. https://doi.org/10.1007/BF02291817

55. Bartlett MS (1954) A note on the multiplying factors for various

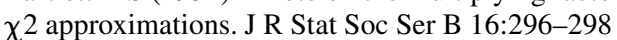

56. Kaiser HF (1960) The application of electronic computers to factor analysis. Educ Psychol Meas 20:141-151. https://doi.org/10. $1177 / 001316446002000116$

57. Cattell RB (1966) The scree test for the number of factors. Multivar Behav Res 1:245-276. https://doi.org/10.1207/s15327906m br0102_10

58. YiLan L, RuTong Z (2015) Clustertend: check the clustering tendency. $\mathrm{R}$ package version 1.4. https://cran.r-project.org/package= clustertend. Accessed 09 Feb 2021

59. Brock G, Pihur V, Datta S, Datta S (2008) clValid: an R package for cluster validation. J Stat Softw 25:1-22. https://doi.org/10. 18637/jss.v025.i04

60. Kuhn M (2020) Caret: classification and regression training. $R$ package version 6.0-86. https://cran.r-project.org/package=caret. Accessed 09 Feb 2021

61. Jolliffe I, Cadima J (2016) Principal component analysis: a review and recent developments. Philos Trans R Soc A Math Phys Eng Sci 374:20150202. https://doi.org/10.1098/rsta.2015.0202

62. Lam M, Lee J, Rapisarda A et al (2018) Longitudinal cognitive changes in young individuals at ultrahigh risk for psychosis. JAMA Psychiat 75:929-939. https://doi.org/10.1001/jamapsychi atry.2018.1668

63. Cobia DJ, Csernansky JG, Wang L (2011) Cortical thickness in neuropsychologically near-normal schizophrenia. Schizophr Res 133:68-76. https://doi.org/10.1016/j.schres.2011.08.017

64. Benassi M, Garofalo S, Ambrosini F et al (2020) Using two-step cluster analysis and latent class cluster analysis to classify the cognitive heterogeneity of cross-diagnostic psychiatric inpatients. Front Psychol 11:1-11. https://doi.org/10.3389/fpsyg.2020.01085

65. Mills JG, Fusar-Poli P, Morgan C et al (2017) People meeting ultra high risk for psychosis criteria in the community. World Psychiatry 16:322-323. https://doi.org/10.1002/wps.20463

66. Schultze-Lutter F, Michel C, Ruhrmann S, Schimmelmann BG (2018) Prevalence and clinical relevance of interview-assessed psychosis-risk symptoms in the young adult community. Psychol Med 48:1167-1178. https://doi.org/10.1017/S0033291717002586

67. Ohi K, Shimada T, Nemoto K et al (2017) Cognitive clustering in schizophrenia patients, their first-degree relatives and healthy subjects is associated with anterior cingulate cortex volume. NeuroImage Clin 16:248-256. https://doi.org/10.1016/j.nicl.2017.08. 008

68. Bora E (2015) Neurodevelopmental origin of cognitive impairment in schizophrenia. Psychol Med 45:1-9. https://doi.org/10. 1017/S0033291714001263

69. Crouse JJ, Chitty KM, Iorfino F et al (2020) Transdiagnostic neurocognitive subgroups and functional course in young people with emerging mental disorders: a cohort study. BJPsych Open 6:1-9. https://doi.org/10.1192/bjo.2020.12

70. Frommann I, Pukrop R, Brinkmeyer J et al (2011) Neuropsychological profiles in different at-risk states of psychosis: executive control impairment in the early-And additional memory dysfunction in the late - Prodromal state. Schizophr Bull 37:861-873. https://doi.org/10.1093/schbul/sbp155

71. Glenthøj LB, Hjorthøj C, Kristensen TD et al (2017) The effect of cognitive remediation in individuals at ultra-high risk for psychosis: a systematic review. npj Schizophr 3:1-8. https://doi.org/ 10.1038/s41537-017-0021-9 